\title{
EARLY IDENTIFICATION OF THE NEUROLOGICAL COMPLICATIONS OF DIABETES MELLITUS
}

\author{
Elcin Huseyn, Research Laboratory of Intelligent Control and Decision-Making Systems in Industry \\ and Economics, Azerbaijan State Oil and Industry University, Baku, Azerbaijan, \\ ORCID ID: https://orcid.org/0000-0001-5965-7419
}

DOI: https://doi.org/10.31435/rsglobal_conf/30032021/7474

Abstract. Diabetes mellitus is still a very common disease in the world and affects the daily lives of patients negatively. Diabetes is also known to be associated with neurological diseases such as peripheral nerve diseases, stroke and dementia. Among these, the most common disease is a peripheral nerve disease, and it has been reported that poor diabetic control increases the risk of development and can be prevented by education of the patients. Vascular dementia is more common in patients with diabetes than Alzheimer's disease, and it is thought that cerebrovascular diseases may be related to cognitive impairment in diabetes. Although the mechanisms by which diabetes affects the brain are not clearly revealed, it is thought that changes in vascular structure, insulin resistance, glucose toxicity, oxidative stress, accumulation of glycation end products, hypoglycemic episodes and amyloid metabolism are effective. The aim of this article is to describe the neurological complications of diabetes and to emphasize the importance of patient education, good diabetes control and early diagnosis in preventing these complications.

Keywords: Biomanufacturing, biomaterial's, differentiation, human bone marrow mesenchymal stem cells.

Introduction. Diabetes mellitus is still a very common disease in the world, negatively affecting the daily lives of patients and continues to be a serious economic burden, especially in countries where obesity is prevalent. It is estimated that diabetes affects 246 million people worldwide and approximately 20-30 million of these patients are affected by symptomatic diabetic polyneuropathy. Considering the increase in obesity rates and the related increase in the prevalence of type 2 diabetes, this number is expected to double in 2030. In young patients with type 1 diabetes, polyneuropathy may occur within a few months from the onset of the disease as a result of poor control of diabetes. Studies show that intense diabetes control reduces the prevalence of clinical neuropathy by $60-69 \%$. Therefore, early diagnosis becomes very important (1-3).

Peripheral neuropathy. Peripheral neuropathy is the most common serious complication of diabetes. This form of neuropathy carries a high risk for pain, atrophic changes, and autonomic dysfunction. Currently, there is no effective treatment for diabetic neuropathy and good glycemic control only minimizes the risk of developing neuropathy in diabetic patients. When diabetic neuropathy occurs, loss of sensation in the feet can be detected, and patient education about the prevention of distal tragic complications is required (1).

Pathophysiology. Diabetic polyneuropathy occurs when there is an imbalance between nerve fiber destruction and repair. Nerve damage formation process mostly affects autonomic and distal sensory fibers. In addition to metabolic factors, ischemic factors and inflammation also have an effect on the development of diabetic neuropathy. While metabolic factors are effective in the long term, inflammation on ischemic nerve damage gains importance in severe forms of focal neuropathy. Ischemia also plays a role in the thickening and hyalinisation of small vessels with a decrease in endometrial oxygenation. Possible mechanisms in the formation of neuropathy are known as oxidative stress, nonenzymatic glycation, polyol and hexosamine pathways, protein kinase c pathway, poly (ADPribose) polymerase, reduction of neurotrophic factors and changes in ion channels, and central excitatory mechanisms. It is accepted that these pathogenic factors play a synergistic role in the development of neuropathy $(2,3)$.

The forms of diabetic neuropathy can be defined according to the anatomical distribution (proximal or distal, symmetric-asymmetric, focal-Multifocal-diffuse), according to clinical course (acute-acute-chronic), characteristic features (painful-painless, sensory, motor or autonomic) or pathophysiologically can be classified (2).

Clinical characteristics of diabetic neuropathy. Chronic distal sensorimotor symmetric polyneuropathy is the most common form of diabetic polyneuropathy is chronic, distal (length 
dependent) symmetrical polyneuropathy and is seen in $75 \%$ of patients with diabetic polyneuropathy. The initial symptoms of length-dependent distal polyneuropathy are numb, burning feet, stinging and electric shock. Symptoms are more pronounced at night and the burning is more severe with contact. Sensory neuropathy is completely silent and can be diagnosed by detailed foot examination, painless trauma or determination of topic changes such as burns or Charcot joint and plantar ulcers. Symptoms and signs in this pattern start from the distal of the lower extremity by first affecting the long nerve endings and gradually progress to the proximal and often affect the distal of the upper extremities after passing the knee level. Short sensory axons are then affected, and with their involvement, neuropathy is seen more proximally and on the anterior aspect of the trunk when distal sensory nerve fibers of the intercostal nerves are affected. Rarely, when the trigeminal nerve is affected, the complaints spread to the head area. If progression is not prevented, it is observed that almost all sensory modalities such as pain and heat disappear. Vibration and joint position sense are rarely affected, suggesting more thick fiber neuropathy. This distribution pattern is called the length dependent pattern. This neuropathy progression is not specific to diabetes and can be seen in alcoholic and amyloid neuropathies. In some patients, weakness and ataxia due to sensory loss draw attention as the main symptoms $(1,2)$.

Lower extremity Achilles reflex cannot be detected on neurological examination. Various degrees of sensory loss are observed, and muscle weakness is more noticeable in the thumb and ankle dorsiflexion. Distal weakness is usually mild and rarely seen.

Sensorimotor neuropathy shows both demyelinating and axonal features in EMG. The earliest and most sensitive findings show up in nerve conduction studies, as a decrease in conduction velocity and amplitude. As neuropathy worsens, loss of sensory response can be seen. In motor conduction studies, a decrease in conduction velocity can be detected even in asymptomatic patients (3).

Selective Fine Fiber Neuropathy. Fine fiber neuropathy is characterized by pain and reduced sensation of heat and pain. Pain is described by patients as a burning, stinging, stabbing or pressing sensation. Patients often experience dysesthesia. Dysesthesia usually occurs in the big toe, but can also spread to the proximals of the lower extremities and upper extremities. Motor power, vibration, proprioception and muscle tension reflexes are preserved in this neuropathy. Nerve conduction studies in EMG are useful in the follow-up of this neuropathy and are not useful for diagnosis. Nerve conduction studies abnormalities occur when thick fibers are affected. Autonomic functions and thermal sensitivity may be useful in testing fine fiber function (3).

Autonomic Neuropathy. Autonomic neuropathy is one of the characteristic features of diabetic neuropathy and can be life threatening. Cardiovascular involvement usually begins with tachycardia at rest. Heart rate can return to normal in the long term, but responses to changing physiological changes are often not achieved. Postural hypotension is perhaps the most life-affecting symptom of autonomic neuropathy because of the syncope it can cause. Postural hypotension can be aggravated by tricyclic antidepressants used in the treatment of diabetic neuropathy and episodes of diarrhea. The strong association of cardiac autonomic neuropathy with silent myocardial ischemia and mortality is well known.

Gastroparesis seen in the gastrointestinal system is generally asymptomatic and may present with swelling from time to time and rarely with vomiting. Hypoglycemia attacks caused by food remaining in the stomach due to gastroparesis pose difficulties in terms of glycemic control. Diabetic diarrhea occurs at night and after meals and is characteristic of being watery. Fecal incontinence may accompany diarrhea.

Frequent urinary tract infections can be seen due to bladder atony and the large residual volume remaining in the bladder after micturition. Impotence is common in male patients. Left untreated, hypoglycemia can disrupt catecholamine release, complicating autonomic neuropathy. Abnormal pupillary responses such as mosses and decreased light reflex are also common findings in diabetes (1).

Focal and Multifocal Neuropathy. In diabetic patients, focal and Multifocal neuropathies are less common than length-dependent diabetic neuropathy. These forms of neuropathy are generally seen after the age of 50 and mainly in patients with type 2 diabetes. Focal neuropathies include cranial nerve involvement, limb and truncal neuropathies, and lower limb proximal diabetic neuropathy. In diabetic patients, development of sensorimotor deficits in one or more nerves, trunks, root or plexus is rare, and nerve biopsy may be necessary to exclude other causes of neuropathy.

Cranial Neuropathy. Acute onset 3rd and 6th nerve paralysis usually occur in elderly patients with poor glycemic control. Oculomotor palsy is the most common cranial neuropathy. In half of the 
cases, it starts with severe pain around the eyes and behind. Oculomotor paralysis develops within a day or two. In diabetic 3rd nerve palsy, the pupil is usually not involved. Spontaneous and complete recovery is observed within months. Multiple cranial nerve paralysis is extremely rare.

Truncal Neuropathy. Truncal neuropathies can be unilateral or bilateral. It can start suddenly and rapidly with pain or dysesthesia, which is the basic clinical picture. Pain often has a radicular distribution and may worsen with touch and at night. Weakness can also be seen in the abdominal muscles.

Isolated involvement of the peripheral nerves in the extremities is extremely rare, except for carpal tunnel syndrome in which the median nerve is trapped. Diabetic polyneuropathy may facilitate the formation of entrapment neuropathies (4).

Proximal Diabetic Neuropathy of the Lower Extremities. This form of diabetic neuropathy, which is less common than distal symmetrical polyneuropathy, draws attention with its acute or subacute, noisy and often asymmetrical onset. The fifth is mostly seen in male patients over women and with weight loss. This syndrome was first described by Bruns in 1890. The disease usually begins with severe pain in one lower limb, mainly in the hip and thigh. Patients have difficulty walking and climbing stairs due to weakness in the quadriceps and iliopsoas muscles. In the early stage of the disease, the patellar reflex disappears due to quadriceps muscle weakness and atrophy. Most cases progress within a few weeks or months and then stabilize, then the pain subsides spontaneously, sometimes abruptly (regardless of glycemic control). The long-term prognosis is good. Nerve conduction studies usually give findings reflecting distal symmetrical neuropathy. Needle electromyography reveals intense partial denervation-reinnervation in the muscles innervated from the legend radix-plexus segments and paraspinal muscles of the symptomatic extremity. CSF protein is generally increased $(1,4)$.

Multifocal Diabetic Neuropathy. Multifocal diabetic neuropathy is observed in a small proportion of patients with diabetes. There may be sequential involvement of the roots, lower extremity, trunk and upper extremity nerves within a few weeks or months, and sometimes a relapsing course may occur. The distal parts of the lower extremities are always involved bilaterally or unilaterally, and most patients may also have proximal deficits. Truncal and upper extremity nerves are less commonly affected (1).

Diabetic Neuropathy Treatment.

Preventive Treatment. Preventing diabetic neuropathy and preventing its complications is the best strategy. Optimum glycemic control reduces the development of peripheral neuropathy, but carries an increased risk of hypoglycemia. Pancreatic transplantation can stabilize neuropathy, but it is not routinely applied yet (1).

Chronic foot wounds of a diabetic patient are the common result of often unnoticed painless traumas, vascular insufficiency, and secondary infections. Patients with diabetic neuropathy should have their examinations repeated frequently, as their prevention is easier than their treatment. Appropriate treatment of developing wounds is very important in terms of preventing limb loss due to gangrene and systemic complications such as sepsis (4).

Symptomatic Treatment. Particular importance are treatments for symptoms of diabetic neuropathy, such as pain, autonomic disorders, and sensory loss. Pain control may be difficult in a length-dependent diabetic neuropathy and focal neuropathies. A combination of carbamazepine, phenytoin, clonazepam, and paracetamol codeine phosphate can be used. Tricyclic antidepressants such as imipramine and amitriptyline are often effective. Tricyclic antidepressants can aggravate postural hypotension. Recently administered duloxetine and pregabalin treatment is also beneficial (1).

Postural hypotension should be treated if symptomatic. Fluorohydrocortisone and midden can be used for this. However, it should be kept in mind that they carry the risk of hypertension (1).

Focal and Multifocal Diabetic Neuropathy Treatment.

Proximal diabetic neuropathy is often very painful, and the pain is often resistant to conventional treatments. In such cases, corticosteroid therapy can be given for a few weeks or months, together with the regulation of glycemic control. It should be kept in mind that the spontaneous prognosis of focal diabetic neuropathies may be good (1).

\section{Central Nervous System Complications of Diabetes.}

Cerebrovascular Diseases. Stroke is 2-6 times more common in patients with diabetes than in non-diabetic patients and plays a role in approximately $25 \%$ of deaths due to diabetes. The metabolic abnormalities of diabetes also negatively affect the existing stroke. Ischemic stroke is more common 
than hemorrhagic stroke in patients with diabetes. It has also been reported that the risk of atrial fibrillation increases by $40 \%$ in diabetes (5-8).

The existence of high risk of stroke in diabetes depends on the complex relationships between various hemodynamic and metabolic components. Metabolic syndrome components such as insulin resistance, central obesity, impaired glucose intolerance and hyperinsulinemia are associated with an increased risk of stroke alone and in combination. Diabetic microangiopathy complications also play an important role in the pathogenesis of stroke, as they increase peripheral resistance and cause atherosclerosis. It is accepted that hemoglobin A1C (Hmg A1C) elevation and postprandial hyperglycemia are risk factors for stroke and increase stroke rates twice $(5,6,8)$.

There is no significant increase in mortality in the first 3 months after acute ischemic stroke in diabetic patients compared to those without diabetes, and it is observed that the mortality rates increase in the first year after stroke. The risk of recurrent stroke also increases in these patients. Diabetes is also associated with increased rates of functional deficits in the long term after stroke (8).

Tight glycemic control is known to prevent microvascular complications, but the effects on macrovascular disease and stroke risks are unclear. Several studies have been conducted comparing strict glycemic control with standard glycemic control in stroke prevention. It has been reported that strict glycemic control does not reduce cardiovascular outcomes and stroke risk in most of them. Tight glycemic control has also been shown to increase the risk of hypoglycemia. Therefore, while providing glucose control, the patient's age, comorbidities and hypoglycemia risk should be taken into account $(6,8)$.

Hyperglycemia is seen in 39-40\% of patients after acute ischemic stroke. Most of these patients do not have a previous history of diabetes. In some patients, hyperglycemia is a marker of previously, but undiagnosed diabetes, while in most patients it is seen as an acute stress response and is called stress hyperglycemia. In this type of hyperglycemia, glucose levels return to normal after discharge. For this reason, $\mathrm{HbAlc}$ levels are useful in distinguishing patients with undiagnosed diabetes, since the high glucose level measured at the time of admission to the hospital cannot be differentiated from stress hyperglycemia and diabetes. Stress hyperglycemia often returns to normal after the acute period. The cause of stress hyperglycemia is the activation of the hypothalamic-pituitary-adrenal axis, the increase in glucocorticoid levels and activation of the sympathetic system (8).

Compared to normoglycemics, patients who are hypoglycemic at admission have a higher mortality risk during hospitalization and after 30 days. This risk is seen as a risk factor independent of other markers in terms of poor prognosis. This relationship between hyperglycemia and poor prognosis is mostly valid in patients with large infarct areas. Moderate hyperglycemia has been found to be associated with better prognosis in patients with lacunar infarction (8).

When the diabetic environment is mentioned, low grade inflammation, endothelial dysfunction, hypercoagulability, dyslipidemia and insulin resistance come to mind. With these factors, new hypotheses have begun to be developed about the pathogenesis of macrovascular complications. 4 different hypotheses have been developed regarding how hyperglycemia affects vascular structure and functions (6).

1. Increased oxidative stress and free radical damage

2. Formation of glycosylation end products

3. Glucose entering the aldose reductase pathway

4. Activation of one or more protein kinase $\mathrm{C}$ isoenzymes

With hyperglycemia, the formation of reactive oxygen metabolites (ROM) and insulinresistant state is toxic to cells. ROMs such as superoxide anions form nitric oxide (NO) toxic peroxynitrite ions and reduce the availability of endothelium-derived NO. In this way, it causes a decrease in endothelial-mediated vasodilation, increased platelet activation, and proliferation and migration of vascular smooth muscles. ROMs also facilitate LDL accumulation in the vascular wall by increasing oxidation. Due to excess glucose, non-enzymatic glycosylation of various proteins and lipoproteins in the vascular wall accelerates the atherosclerotic process, which increases the uptake and oxidation of LDL in the cell and causes foam cell formation (6).

In the 2014 AHA Guideline, diabetes screening with a fasting glucose level, OGTT or HmgA1C is recommended in all patients after an acute cerebrovascular event (9). Studies have reported that hyperglycemia detected during admission and hospitalization is associated with poor clinical results and larger infarct volume. There are pilot studies showing that it is safe to reduce glucose aggressively with insulin therapy in the acute phase. However, it is not yet clear whether 
keeping glucose levels at certain levels has a positive effect on the results. The biggest side effect of aggressive hyperglycemia treatment is hypoglycemia. Therefore, differential diagnosis of stress hyperglycemia and diabetes in acute ischemic stroke, treatment with hyperglycemia, but attention should be paid to recurrent hypoglycemia during treatment. The recommendation of the American Diabetes Association is to keep glucose levels between $140-180 \mathrm{mg} /$ deal in all inpatients $(6,8)$.

Dementia. Dementia is a syndrome that affects patients' memory, thinking, behavior and daily life activities. Alzheimer's disease is the most common dementia subtype, and it is considered a primary neurodegenerative disease characterized by neuritic plaques and neurofibrillary tangles. Vascular dementia is the second most common dementia syndrome and is seen after stroke with small vessel disease and chronic brain ischemia. According to the data of the World Health Organization, the number of patients diagnosed with dementia was 35.6 million in 2012, while this number is expected to increase to 65.7 million in 2030 and 115.4 million in 2050. Therefore, dementia emerges as an important public health problem. Because of its increasing prevalence and affecting many systems, diabetes is also seen as an important public health problem (10).

It has been reported in many epidemiological studies that patients with diabetes are at risk for cognitive impairment and dementia. However, in the studies, the subtypes of dementia could not be clearly revealed due to the mixed type of neurodegenerative and vascular pathologies of the patients and therefore contradictory results were obtained. Therefore, the true link between diabetes and dementia is still controversial, and the factors of cognitive decline are not yet clearly understood. In some population-based studies, it has been revealed that there is a relationship between diabetes and dementia. In a meta-analysis examining 18 studies, it was shown that diabetes increased all types of dementia on average 1.7 times (11). Looking at the subtypes of dementia, it is seen that diabetes is a significant risk factor for both Alzheimer's disease and vascular dementia. Some case-control studies have advocated a negative relationship between Type 2 diabetes and Alzheimer's disease and advocated a possible protective effect of high glucose levels on the brain (10-13).

Changes in the hippocampus, amygdala and medial temporal lobe are observed in cranial MRI in the early period in Alzheimer's disease. The decrease in hippocampal and amygdala volume in cranial MRI provides us important information in terms of the degree of Alzheimer's neuropathology. In the Rotterdam study, it was shown that the hippocampus and amygdala volumes in cranial MRIs of diabetic patients decreased compared to patients without diabetes. The relationship between diabetic factors and Alzheimer's disease neuropathology has also been demonstrated by pathological studies. In Hisayama's study, it was shown that after 2 hours OGTT, neuritic plaques increased and this relationship was more pronounced in APOEe4 allele carriers. In the light of these findings, it can be thought that hyperinsulinemia and insulin resistance worsen neuropathologies in Alzheimer's disease, especially in APOEe4 allele carriers.

The mechanism of the relationship between diabetes and dementia has not yet been clearly elucidated. However, considering that demographic and socioeconomic factors and genetic factors trigger different pathological processes in both diseases, as well as cardiovascular risk factors associated with diabetes, glucostoxicity, insulin resistance and inflammation, this relationship can be considered to be multifactorial (12).

Hyperglycemia can cause worsening in memory and attention. Chronic hyperglycemia may cause cognitive impairment and synaptic plasticity pathologies. With glucostoxicity, oxidative stress and the accumulation of glycosylation end products occur and after the formation of vascular damage, neurodegeneration begins in the brain. However, the role of strict glycemic control in the prevention of cognitive impairment is still controversial $(14,15)$.

Studies have reported that hypoglycemia that develops in patients with Type 2 Diabetes is also effective in cognitive breakdown. It has been shown that the rate of cognitive impairment increases 1. 5-2 times in patients with recurrent hypoglycemia. It has also been reported that fibrinogen formation, together with neuronal death and platelet aggregation, can accelerate the cognitive degradation process in severe hypoglycemia (16).

Insulin resistance and hyperinsulinemia are partially associated with cognitive impairment due to vascular disease. There is evidence that insulin and insulin receptors play an important role in cognitive performance through modification of excitatory and inhibitory postsynaptic receptor activity and activation of specific signaling pathways. Insulin receptors are present in many parts of the brain, 
including the hippocampus and cortex. It has been found that prolonged hyperinsulinemia impairs the insulin response due to the decrease in the blood-brain barrier and the number of insulin receptors in the brain, and inhibits insulin passage to the CSF and brain tissues. These changes create defects in learning and memory formation. Impairment of insulin sensitivity of the brain has been detected in Alzheimer's patients compared to healthy individuals. The insulin degrading enzyme breaks down both insulin and amyloid. In the case of high plasma insulin levels, destruction enzymes compete, reducing amyloid beta protein degradation and provoking amyloid accumulation $(16,17)$.

Since the pathophysiological findings of dementia appear long before the symptoms, it may be beneficial to control the risk factors that may cause this, especially diabetes, in the early period.

In conclusion, diabetes is a common disease with serious complications affecting the nervous system. Early diagnosis and treatment in preventing complications

Is of great importance.

\section{REFERENCES}

1. Said G. Diabetic neuropathy-A Review. Nat Clin Prac Neurol 2007; 3:331-340. http://dx.doi.org/10.1038/ncpneuro0504

2. Albers JW. Diabetic Neuropathy: Mechanisms, Emerging Treatments and Subtypes. Curr Neurol NeurosciRep 2014;14:473. http://dx.doi.org/10.1007/s11910-014-0473-5

3. Charnogursky G. Neurological Complications of diabetes. Curr Neurol Neurosci Rep 2014; 14:457. http://dx.doi.org/10.1007/s11910-014-0457-5

4. Emre M. Neurology, Issue 1:2. 2013;535-540.

5. Zarko S. Amınoff M. Neurology and General Medicine. 4.Issue (395-405).

6. Idris I, Thomson, GA, Sharma JC. Diabetes mellitus and stroke. Int J Clin Pract 2006;60(1):48-56. http://dx.doi.org/10.1111/j.1368-5031.2006.00682.x

7. Rohit R. Das, MD, Seshadri S. Diabetes and Stroke. DM Current Cardiovascular Risk Reports 2009;3:35-41.

8. Merel JA, Biessels GJ. Diabetes, hyperglycaemia, and acute ischaemic stroke, Lancet Neurol 2012;11:261271. http://dx.doi.org/10.1016/S1474-4422(12)70005-4

9. Kernan WN, Ovbiagele B, Black HR. Guidelines for the Prevention of Stroke in patients with stroke and Transient Ischemic Attack. DOI:10.1161/STR.000000000000002

10. World Health Organization and Alzheimer's Disease International. Dementia. A public health priority. Available at: http://www.who.int/mental health/publications/dementia report 2012/en/. Accessed 18 Aug 2013.

11. Biessels GJ, Staekenborg S, Brunner E, Brayne C, Scheltens P. Risk of dementia in diabetes mellitus: a systematic review. Lancet Neurol 2006;5:64-74. http://dx.doi.org/10.1016/S1474-4422(05)70284-2

12. Ninomiya T. Diabetes mellitus and dementia: Curr Diab Rep 2014;14:487. http://dx.doi.org/10.1007/s11892-014-0487-z

13. Stewart R. Cardiovascular factors in Alzheimer's disease. J Neurol Neurosurg Psychiatry 1998;65:143-147. http://dx.doi.org/10.1136/jnnp.65.2.143

14. Launer LJ, Miller ME, Williamson JD, et al. Effects of intensive glucose lowering on brain structure and function in people with type 2 diabetes (accordmind): a randomized open study. Lancet Neurol 2011;10:969-972 http://dx.doi.org/10.1016/S1474-4422(11)70188-0

15. de Galan BE, Zoungas S, Chalmers J, et al. ADVANCE Collaborative Group. Cognitive function and risks of cardiovascular disease and hypoglycaemia in patients with type 2 diabetes: the Action in Diabetes and Vascular Disease: Preterax and Diamicron Modified Release Controlled Evaluation (ADVANCE) trial. Diabetologia 2009;52:2328-2336. http://dx.doi.org/10.1007/s00125-009-1484-7

16. Zhao WQ, Alkon DL. Role of insulinandinsulinreceptor in learning and memory. Mol Cell Endocrinol. 2001;177:125-134. http://dx.doi.org/10.1016/S0303-7207(01)00455-5

17. Neumann KF, Rojo L, et al. Insulin resistance and Alzheimer's disease: molecular links\&clinical implications. Curr Alzheimer Res 2008;5:438-447. http://dx.doi.org/10.2174/156720508785908919 